Method: Little evidence-based literature is available concerning the mean ratio of T1:T2:T3:T4 resulting from triage of real casualties in the CEP-missions in Germany. A review of $528 \mathrm{CEP}$-missions from six rescue districts was conducted to evaluate the average number of patients in CEP missions, and to make an estimate of the Severity Index (SI) of the missions based on the results, according to the four categories of triage (red, yellow, green, black). As calculated from De Boers Formula, $\mathrm{SI}=(\mathrm{T} 1+\mathrm{T} 2) / \mathrm{T} 3$, the mean SI of a subgroup of the Bavarian CEP missions (January 1996-August 2004) was determined in order to obtain an impression of what has been occurring relative to mass-casualties in the country, and to develop an estimate of the potential for major hazards in the reported area and time corridor.

Results: In the 528 CEP missions reviewed, a total of 3,136 patients were treated. Another 3,080 people had been involved without requiring more help than advised (bystanders involved). The red:yellow:green:black ratio resulting from the $>500$ missions with a average of six patients was: $27.2: 20.6: 51.3: 2.6(\%)$, which can be simplified to 1:1:2:2.5 (\%). The average of the SIs resulting from these figures was 0.93 for the $>500$ missions.

Conclusion: The CEP system implemented is working in this area, since the average of the SIs is below 1.0. The main hazards causing these CEP-missions, according to the Utstein Style, will be discussed according to the incident frequency. Correlating studies with other regions have been planned to verify that the average incidence for CEPmissions is about one mission per month in an area with about $1,000,000$ inhabitants.

Keywords: Bavaria; coordinating emergency physician (CEP); Germany; missions; severity index; triage

Prebosp Disast Med 2005;20(2):s25-s26

\section{Prehospital Emergency Medical Service Systems in Portugal}

N. Pereira; $M$. Soares-Oliveira

Instituto Nacional de Emergência Médica (INEM; National Institute of Emergency Medicine), Portugal

In Portugal, the prehospital emergency medical service system (PEMSS) is coordinated by the National Institute of Emergency Medicine (INEM). The INEM is a governmental organization directly dependent on the Ministry of Health. The PEMSS currently is accessible to the majority of the population. Portuguese prehospital emergency systems engage doctors in every step of the chain of care.

The aim of this presentation is to describe the Portuguese system and to overview the involvement of medical services in the system.

The INEM is divided functionally into four regional centers (Porto, Coimbra, Lisboa, and Faro), each with a dispatch center (CODU), which runs the PEMSS. Every 1-1-2 (emergency) call concerning health problems is transferred to to CODU. There is a medical doctor in the CODU 24 hours a day. In each region, there are hospital-based rapid intervention vehicles (VMER), staffed with a medical doctor and nurse. Approximately ten million people (83\% of the population) have direct access to the PEMSS. Altogether, the four CODU receive a mean of 2,750 calls a day. There are 27 VMER in the country (nine in Porto, eight in Coimbra, eight in Lisboa, and two in Faro). Each VMER is equipped with advanced life support (adult and pediatric) and trauma life support equipment. The team is composed of a nurse and a medical doctor with education and experience in emergency medicine. The VMER had 1,906 services per month in the first six months of 2004 . The CODU also dispatches all the ambulances of the enclosed area. Between January and July 2004, the CODU dispatched 188,220 ambuilances. There are also two medical helicopters, one in Porto and one in Lisboa. The helicopters are designed to assist victims in the field and/or to transfer emergency patients between hospitals. The composition of the team and the available equipment are similar to the VMER. Besides dispatching, the CODU also has the obligation to direct victims to the appropriate, available hospital. Furthermore, the hospital preparation to take delivery of the victim should be triggered by the CODU. Keywords: dispatch center (CODU); National Institute of Emergency Medicine (INEM); Portugal; prehospital emergency medical service system (PEMSS); rapid intervention vehicles (VMER)

Prehosp Disast Med 2005;20(2):s26

\section{Free Papers Theme 9: Emergency Medical Services System Design- Specific Issues} Infrastructure of a Level-One Trauma Center for
Mass-Casualty Incident Due to Conventional and
Unconventional Weapons: Sharing Drill among
National Agencies

O. Benin-Goren; P. Gedalia; A. Lior; T. Shamis

Tel Aviv Sourasky Medical Center, Israel

Mass-casualty incidents (MCIs) create challenges for medical response in the field and in hospitals. MCIs due to weapons of mass destruction and disasters involving terrorism require cooperation among all agencies involved with $\mathrm{MCls}$ (e.g., police, firefighters, emergency medical services, Army Medical Corps, Home Front Command, and the Ministry of Health). A well-organized incident command system can help all involving agencies provide the best quality of services in order to save lives in such an event.

Unfortunately, the security situation in Israel has provided training in how to deal with MCIs. Tel Aviv Sourasky Medical Center (TASMC) had a significant experience with the management of MCIs. Nevertheless, Israel's experience is related to $\mathrm{MCI}$ caused by conventional weapons. Yet, since the real threat also includes unconventional weapons of mass destruction, hospital preparedness always included decontamination and medical care for chemical weapons.

In the November $2004 \mathrm{MCI}$ Drill, which involved conventional and unconventional weapons took place in the area of Tel Aviv. It was the first MCI Drill related to the compound situation that was performed in Israel and 
involved all agencies taking part in such an event. Five medical centers took part in the drill. Four of them were trained and evaluated at the command function level, one of the hospitals; TASMC, additionally was trained and evaluated, according to its function at the command level, on the quality of medical care, hospital infrastructure and capacity, staff deployment, patient observation, and secondary evacuation due to hospital incapacity.

This work will share our experience with the infrastructure of one of the biggest medical centers in Israel prepare for compound MCIs. It also includes the training program, staff deployment and the establishment of guidelines for in-hospital site function and medical protocols. It also includes the results and evaluation of the drill performances among all the cooperating agencies.

Keywords: Israel; mass-casualty incident; trauma center Prehosp Disast Med 2005;20(2):s26-s27

\section{Emergency Treatment Models of Trauma in China Z. Wang; ${ }^{1}$ W. Wang ${ }^{2}$ \\ 1. Research Institute of Surgery, China \\ 2. China}

Up to $35 \%$ of the deaths from severe trauma can be prevented if treatment is appropriate and timely. The preventability and impact of trauma care systems on disability is predicted to be similar.

In the early 1980 s in China, emergency medical services (EMS) began to develop rapidly and many emergency medical centers or stations were established. In 1986, the National Department of Public Health and the National Department of Post-Telecommunication jointly declared 1-2-0 as the nationwide emergency telephone number. In a city of $>400,000$ people, emergency medical organizations must be established. To date, there are four emergency medical models in China.

In the Beijing model, centers consist of a complete set of advanced equipment and units, including a garage, intensive care unit (ICU) with 28 beds, hyperbaric oxygen chamber, CT scan, operating room, emergency department, first-aid room, pharmacy, dialysis room, and blood bank.

In the Shanghai model, there is a municipal emergency medical service center responsible for prehospital emergency treatment of the whole city. Intra-hospital emergency treatment and the ICU are controlled with different levels by the hospital emergency departments. Most of the $>100$ emergency centers in China follow this model.

In the Guangzhou model, there is a municipal emergency medical communication-conducting center. Prehospital emergency treatment is provided by the emergency department of various hospitals which are responsible for a given district or area.

In the Chongqing model, the emergency center is attached to a modern and advanced hospital. In fact, prehospital emergency has become a part of the hospital. Even though this model is different, its function is the same, i.e., providing first aid on the scene and giving the best life support to patients with severe trauma.

Keywords: China; emergency center; models; prehospital; treatment Prehosp Disass Med 2005:20(2):s27
Citizen and Emergency Responder Shared Values: Enabling Mutual Disaster Management Performance A. Drabczyk

USA

An emergent paradigm in the United States post-11 September 2001 is the need for increased recruitment and training of citizen responder volunteers to work in tandem with professional emergency responders in order to expand the capacities for damage mitigation, and disaster, preparedness, response, and recovery. A Community Emergency Response Team (CERT) served as the model for this study of a citizen and professional partnership.

This research sought improved knowledge regarding the shared values of the citizen and emergency responders, which motivate and sustain their mutual association. The CERT participants were the sole entities that could identify, acknowledge, and validate the aspects of their mutual experience that had value. A participative action research (PAR) method entitled Appreciative Inquiry (AI) was utilized to investigate citizen responder values, emergency responder values, and shared values of the CERT partners. Professor David Cooperrider of Case Western Reserve University in Cleveland, Ohio, USA, established AI as a viable PAR in the 1980s. AI encourages story-telling of best moments shared by a cohort, and themes emerge from the stories.

Positive and progressive conversations surrounded the four questions posed to research participants: (1) Describe a peak experience or high point with your CERT. This would be a time when you were most alive and engaged; (2) Without being modest, what is it that you most value about yourself, the nature of your work, and your CERT?; (3) What are the core factors that give life to your CERT, without which the CERT would cease to exist?; and (4) What are three wishes you have to enhance the health and vitality of your CERT? The AI questions evoked affirmations of CERT past, present, and future experiences, and a total of 94 values were identified.

Findings from this study suggest that both the citizen and emergency responder share four value groupings identified as: (1) functioning/task-related; (2) cohesiveness/relationship; (3) development/change-related; and (4) stabili$\mathrm{ty} / \mathrm{status}$ quo. Recommendations from the study will enable improved recruitment, training, and sustainability of volunteer citizen responders, and enhanced job satisfaction of professional emergency responders. An improved knowledgebase and understanding of shared values of a CERT should increase municipal disaster management capability.

Keywords: Appreciated Inquiry; citizens; Community Emergency Response Team (CERT); disaster management; job satisfaction; responders; values; volunteers

Prebosp Disast Med 2005;20(2):s27 